\title{
AUTISM DIAGNOSTICS BY CENTERLINE-BASED SHAPE ANALYSIS OF THE CORPUS CALLOSUM
}

\author{
A. Elnakib ${ }^{1}$, M. F. Casanova ${ }^{2}$, G. Gimel'farb ${ }^{3}, A$. E. Switala ${ }^{2}$, and A. El-Baz ${ }^{1 *}$ \\ ${ }^{1}$ BioImaging Laboratory, Bioengineering Department, University of Louisville, Louisville, KY, USA. \\ 2 Department of Psychiatry and Behavioral Science, University of Louisville, Louisville, KY, USA. \\ ${ }^{3}$ Department of Computer Science, University of Auckland, Auckland, New Zealand.
}

\begin{abstract}
Autism severely impairs personal behavior and communication skills, so that improved diagnostic methods are called for. Neuropathological studies have revealed abnormal anatomy of the Corpus Callosum (CC) in autistic brains. We explore a possibility of distinguishing between autistic and normal (control) brains by quantitative CC shape analysis in the 3D magnetic resonance images (MRI). Our approach consists of the three steps: (i) segmenting the CC from a given 3D MRI using the learned CC shape and visual appearance; (ii) extracting a centerline of the CC; and (iii) classifying the subject as autistic or normal based on the estimated length of the centerline of the $\mathrm{CC}$ using a k-Nearest neighbor classifier. Experiments revealed significant differences (at the $95 \%$ confidence level) between the $\mathrm{CC}$ centerlines for 17 normal and 17 autistic subjects. Our initial classification suggests the proposed centerlinebased shape analysis of the CC is a promising supplement to the current autism diagnostics.
\end{abstract}

Index Terms - Autism, Diagnostics, Shape analysis, Corpus callosum, Segmentation.

\section{INTRODUCTION}

Autistic Spectrum Disorder (ASD), or autism, is a complex neurological disability characterized by qualitative abnormalities in behavior and higher cognitive functions [1]. It typically appears during the first three years of life and impacts development of social interaction and communication skills. According to the Centers for Disease Control and Prevention (CDC, 2006), about 1 in 110 American children fall somewhere in the autistic spectrum. Although the cause of autism is still largely not clear, researchers have suggested that genetic, developmental, and environmental factors may be the cause or the predisposing effects towards developing autism [2]. Multiple studies during the past decade have revealed that different brain structures are involved in the abnormal neuro-development associated with autism. For example, MRI studies have shown an increased volume in cerebellar white matter of young children with autism relative to controls [3]. Also, cortical grey matter enlargement, particularly in the frontal and temporal lobes, is another abnormal feature of the brain in autistic patients [4].

This paper develops a new framework for analyzing the surface of CC for normal and autistic subjects. The goal is to identify whether or not the $\mathrm{CC}$ involved in the abnormal neural development is associated with autism. The CC is the largest fiber bundle

${ }^{*}$ Corresponding Author:- Tel: (502) 852-5092, Fax: (502) 852-6806, E-mail: aselba01@louisville.edu connecting the left and the right cerebral hemispheres in the human brain. Since the higher cognitive functions of the brain are highly affected by the impaired communication between the hemispheres, several studies [5-10] have proposed to analyze the CC for autistic subjects. In [5-7], the CC had been traced from the midsagittal MRI slice. Statistical difference analysis was applied to find out which part in the CC contributes significantly to identification of autistic brains. Chung et al. [8] applied a voxel based morphometry approach using a freely available public domain software package (i.e., SPM software-http://www.fil.ion.ucl.ac.uk/spm/) to spatially normalize the midsagittal MRI slice to a common stereotactic space in order to segment the CC and localize the CC subregions that are related to autism. To cover more CC anatomy, studies $[9,10]$ account not only for the midsagittal slice but also for four adjacent slices on both sides. He et al. [9] traced the CC from the nine slices based on a semi automated active contour methodology. A contour stitching technique was applied to create the 3D CC surfaces for each subject. Statistical difference analysis was applied to the signed distance map from each subject surface to a template. Instead of using a signed distance map metric, Vidal et al. [10] utilized the CC thickness - the distance between uniformly spaced points on the $\mathrm{CC}$ surface to the $\mathrm{CC}$ medial line (i.e., the average curve between superior and inferior $\mathrm{CC}$ boundaries) - to localize regions of callosal thinning in autism.

To identify whether the abnormal neural development of the CC is associated with autism, we compare directly the extracted centerline that describes the 3D surfaces of the $\mathrm{CC}$ for normal and autistic subjects. To the best of our knowledge, all the previous works have focused on analyzing either the 2D midsagittal cross-section of the $\mathrm{CC}$ or the midsagittal slice along with four adjacent slices on both sides. Unfortunately, this is insufficient for describing the whole shape of the CC and is the main motivation behind our approach. Section 2 below overviews in brief our CC segmentation, from all the slices that the $\mathrm{CC}$ appears in, using a learned $\mathrm{CC}$ shape prior and a learnable joint Markov-Gibbs random field (MGRF) model of 3D MRI and 3D object-background maps. Section 3 shows the Eikonalbased centerline extraction for the segmented CC. In contrast to the known 2D solutions (e.g. [11]), the proposed process evolves in the 3D space in order to detect 3D points of the maximal curvature. Experimental results and conclusions are given in Section 4.

\section{SEGMENTATION OF CORPUS CALLOSUM USING A SHAPE MODEL AND A JOINT MGRF MODEL OF 3D MRI}

Let $\mathbf{Q}=\{0, \ldots, Q-1\}, \mathbf{L}=\{\mathrm{ob}, \mathrm{bg}\}$, and $\mathbf{U}=[0,1]$ be a set of $Q$ integer gray levels, a set of object ("ob") and background ("bg") labels, and a unit interval, respectively. Let a $3 \mathrm{D}$ arithmetic grid $\mathbf{R}=\{(x, y, z): x=0,1, \ldots, X-1 ; y=0,1, \ldots, Y-1 ; z=$ 
$0,1, \ldots, Z-1\}$ support grayscale MRI $\mathbf{g}: \mathbf{R} \rightarrow \mathbf{Q}$, their binary region maps $\mathbf{m}: \mathbf{R} \rightarrow \mathbf{L}$, and probabilistic shape model $\mathbf{s}: \mathbf{R} \rightarrow \mathbf{U}$. The shape model allows for registering (aligning) 3D brain MRI. The co-registered 3D MRI and their region maps are modeled with a joint MGRF specified by a probability distribution $P(\mathbf{g}, \mathbf{s}, \mathbf{m})=P(\mathbf{g} \mid \mathbf{m}) \mathbf{P}(\mathbf{s} \mid \mathbf{m}) P(\mathbf{m})$; where $P(\mathbf{m})$ is an unconditional Gibbs distribution of co-registered region maps, $P(\mathbf{g} \mid \mathbf{m})$ is a conditional distribution of the MRI signals given the map, and $P(\mathbf{s} \mid \mathbf{m})$ is a conditional distribution of the prior shape of the CC given the map.

As shown in Fig. 1, we focus on accurate identification of spatial voxel interactions in $P(\mathbf{m})$, voxel-wise distributions of intensities in $P(\mathbf{g} \mid \mathbf{m})$, and prior distribution of the shape of the CC in $P(\mathbf{s} \mid \mathbf{m})$ for co-aligned 3D MR images. The probabilistic 3D shape model $\mathbf{s}$ is learned from a training set of manually segmented and co-aligned images. To perform the initial CC segmentation, every given MRI is aligned to one of the training images. The shape model provides the voxel-wise object and background probabilities being used, together with the conditional image intensity model $P(\mathbf{g} \mid \mathbf{m})$, to build an initial region map. The final Bayesian segmentation is performed using the identified joint MGRF model of the MRI and region maps.

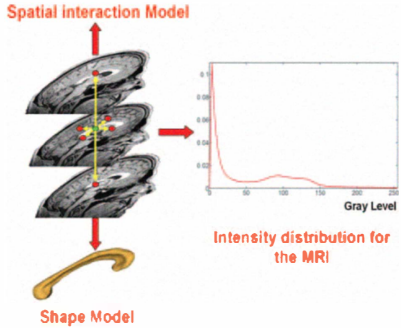

Fig. 1. The joint Markov-Gibbs random field model of 3D MR images.

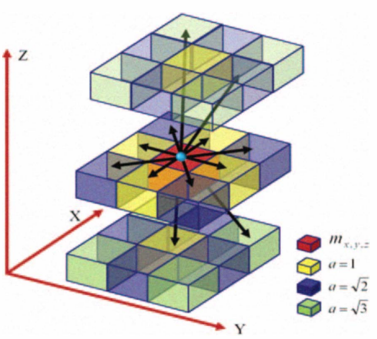

Fig. 2. $3 \mathrm{D} 2^{\text {nd }}$ order $\mathrm{MRF}$ neighborhood system. The reference voxel is shown in red.

\subsection{Spatial voxel interaction in the $\mathbf{C C}$}

For a more accurate segmentation, spatially homogeneous interactions between the region labels are modeled with the 3D extension of the popular Potts model (the MGRF with the nearest 26-voxel neighborhood, Fig. 2) having bi-valued Gibbs potentials, depending only on whether the nearest pairs of labels are equal or not.

The 26-neighborhood, $\mathbf{N}_{a}$, has three types of symmetric pairwise interactions specified by the absolute distance $a \in \mathbf{A}=$ $\{1, \sqrt{2}, \sqrt{3}\}$ between two voxels in the same and adjacent MRI slices, as shown in Fig. 2.

To identify the MGRF model, approximate analytical maximum likelihood estimate of the 3D Gibbs potentials, $V_{a}$, eq,$V_{a}$, ne are derived in line with [12]: $V_{a, \mathrm{eq}}=-V_{a, \mathrm{ne}}=2\left(f_{\mathrm{eq}}(\mathbf{m})-1 / 2\right)$ where $f_{a, \text { eq }}(\mathbf{m})$ denotes the relative frequency of the equal label pairs in the equivalent voxel pairs $\{((x, y, z),(x+\xi, y+\eta, z+\kappa))$ : $\left.(x, y, z) \in \mathbf{R} ;(x+\xi, y+\eta, z+\kappa) \in \mathbf{R} ;(\xi, \eta, \kappa) \in \mathbf{N}_{a}\right\}$. of a region map $m$ of a given MRI aligned in accord with the prior shape model.

\subsection{Conditional intensity model for the 3D MRI}

Just as in $[12,13]$, a 3D MRI, given a region map, is modeled with a simple conditionally independent random field of voxel intensities:

$$
P(\mathbf{g} \mid \mathbf{m})=\prod_{(x, y, z) \in \mathbf{R}} p_{m_{x, y, z}}\left(\mathbf{g}_{x, y, z}\right)
$$

where the voxel-wise probability distributions $p_{\lambda}=\left[p_{\lambda}(q): q \epsilon\right.$ Q]; $\lambda \in \mathbf{L}$, for the CC and its background are estimated during the segmentation. To separate $p_{0}$ and $p_{1}$, the mixed empirical distribution of all voxel intensities is closely approximated with a linear combination of discrete Gaussians (LCDG) with two dominant modes related to the object (i.e. the $\mathrm{CC}$ ) and background, respectively. The LCDG including numbers of its positive and negative terms is obtained with our previous Expectation-Maximizationbased algorithm introduced in $[12,13]$.

\subsection{Probabilistic model of the $\mathrm{CC}$ shape}

Most of the recent works on image segmentation use level set based representations of shapes: an individual shape is outlined by a set of boundary pixels (or voxels) at the zero level of a certain distance function, and a given shape is approximated with the closest linear combination of the training shapes. The main drawback of this representation is that the space of signed distances is not closed with respect to linear operations. As a result, linear combinations of the distance functions may relate to invalid or even physically impossible boundaries.

To circumvent this limitation, the probabilistic 3D CC shape model $\mathbf{s}: \mathbf{R} \rightarrow \mathbf{U}$ where $s(x, y, z)$ is the empirical probability that the voxel $(x, y, z)$ belongs to the $\mathrm{CC}$ is learned from the coregistered training MRI. Such a prior is constructed by:

1. Co-align the training set of MRI using a rigid 3D registration with mutual information as a similarity measure [14] (Fig. 3(a;b))

2. Manually segment the CCs from the aligned set (Fig. 3(c)).

3. Estimate the voxel-wise probabilities $s(x, y, z)$ by counting how many times the voxel $(x, y, z)$ was segmented as the CC (Fig. 3(d)).
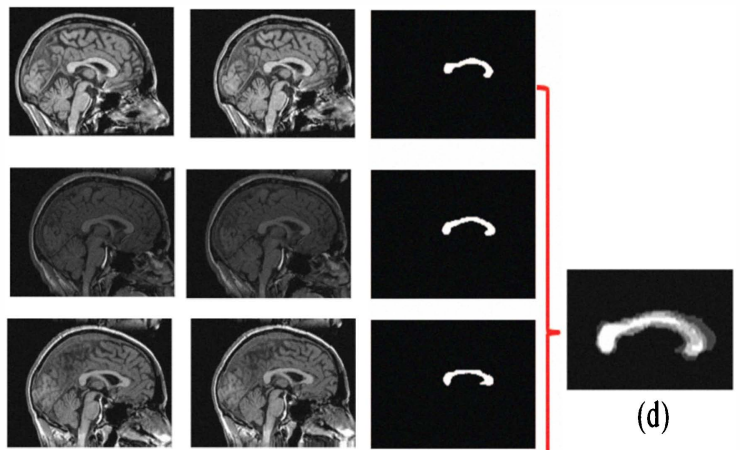

(d)

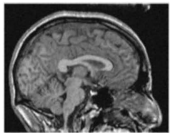

(a)

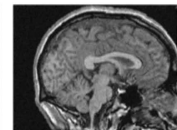

(b)

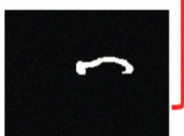

(c)
Fig. 3. Shape reconstruction (2D illustrations): database samples (a), affine mutual information based registration (b), manual segmentation (c), and the estimated prior CC shape (d).

\subsection{Segmentation algorithm}

In total, the proposed $\mathrm{CC}$ segmentation is obtained by the following processing steps:

1. Perform an affine alignment of a given 3D MRI to an arbitrary prototype $\mathrm{CC}$ from the training set using mutual information as a similarity measure. 
2. Estimate the conditional intensity model $P(\mathbf{g} \mid \mathbf{m})$ by identifying the bimodal LCDG.

3. Use the intensity model found and the learned probabilistic shape model to perform an initial segmentation of the CC, i.e. to form an initial region map.

4. Use the initial region map to identify the MGRF model $P(\mathbf{m})$ of region maps and update the conditional intensity model $P(\mathbf{g} \mid \mathbf{m})$.

5. Perform the final Bayesian segmentation of the $\mathrm{CC}$ in accord with the updated joint MGRF model $P(\mathbf{g}, \mathbf{m})$.

\section{CENTERLINE EXTRACTION FROM THE CC}

The problem of extracting the centerline connecting splenium (e.g. the point $A$ in Fig. 4(a)) with rostrum (the point $B$ ) can be formulated as a minimum-cost problem: find the path that minimizes the cumulative cost of traveling from the starting point $A$ to the destination $B$. As defined in [15], if $W(x, y, z)$ is a cost function at any location $(x, y, z)$ inside the $\mathrm{CC}$ then the minimum cumulative cost at the location $B=\left(x^{\prime}, y^{\prime}, z^{\prime}\right)$ is

$$
T(B)=\min _{C_{A B}} \int_{0}^{L} W(C(l)) d l
$$

where $L$ is the path length and $C_{A B}$ is a set of all possible paths linking $A$ to $B$ such that $C(0)=A$ and $C(L)=B$ are the starting and ending points of each path $C(l) \in C_{A B}$. The minimum cost path solving Eq. (1) also satisfies the solution of the Eikonal equation:

$$
|\nabla T(x, y, z)| F(x, y, z)=1
$$

where $T(x, y, z)$ is the time at which the front evolving from the point $A$ crosses the point $(x, y, z)$, and $F(x, y, z)$ is the speed function.

We propose a new algorithm to extract the centerline of the 3D CC based on solving Eq. (2):

1. Find the boundary of the segmented $\mathrm{CC}$ by estimating its $3 \mathrm{D}$ edges (see Fig. 4(b)).

2. Find the normalized minimum Euclidian distance $D(x, y, z)$ from every inner CC point $(x, y, z)$ to the CC boundary (Fig. 4(c)) by solving Eq. (2) using the fast marching level sets at the unit speed function, $F(x, y, z)=1$ [16].

3. Extract points located on the 3D centerline of the $\mathrm{CC}$ as follows:

(a) Pick any splenium point as a starting point, $A$.

(b) Propagate an orthogonal wave from the point $A$ by solving Eq. (2) using the fast marching level sets at the speed function $F(x, y, z)=\exp (-D(x, y, z))$ (Fig. 4(d)).

(c) Track the point with the maximum curvature for each propagating wave front (Fig. 4(e,f)), this point being considered at any time as corresponding to the starting point $A$.

(d) The point $B$ at which the maximum curvature point of the propagating wave hits rostrum of the $\mathrm{CC}$ is selected as the end point of the centerline.
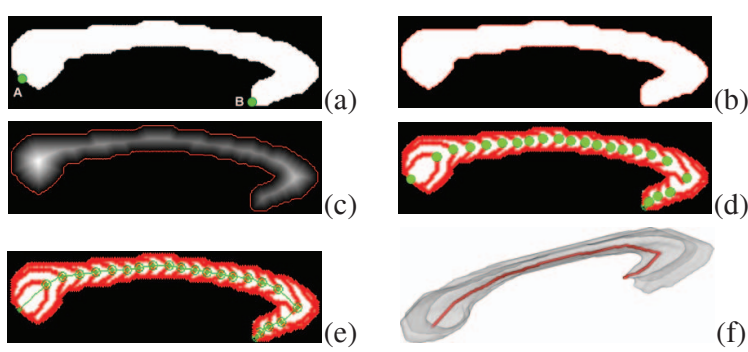

(f)

Fig. 4. Steps of the proposed centerline algorithm illustrated by the saggital 2D cross-sections of the 3D CC (a), estimated 3D CC edges (b), normalized distance map (c), orthogonal wave propagated from the point $A(\mathrm{~d})$, extracted centerline (e), and the 3D visualization of the extracted centerline (f).

\section{EXPERIMENTAL RESULTS AND CONCLUSION}

The proposed approach has been tested on in-vivo data collected from 17 autistic subjects aged 16 to 22 years and a group of 17 controls who match for gender, age, educational level, socioeconomic background, handedness, and general intelligence. All the subjects are physically healthy and free of history of neurological diseases and head injury. Briefly, all the subjects have exactly the same psychiatric conditions. All images were acquired with the same 1.5 T MRI scanner (GE, Milwaukee, Wisconsin) with voxel resolution $1.0 \times 1.0 \times 1.25 \mathrm{~mm}^{3}$ using a T1 weighted imaging sequence protocol. The "ground truth" diagnosis to evaluate the classification accuracy for each patient was given by clinicians.

3D CC Segmentation: The results of the proposed CC segmentation algorithm is illustrated in Fig. 5. For the segmentation error calculation, we determine the true positive $(T P)$, true negative $(T N)$, false positive $(F P)$, and false negative $(F N)$ segmentation. $T P$ was defined as an overlapping area (the number of pixels) between the segmented region, $S e g$, and a ground truth region, $G T$, represented by $T P=S e g \cap G T$. $F N$ was represented by $F N=$ $G T-S e g \cap G T$. FP was represented by $F P=S e g-S e g \cap G T$. $T N$ was represented by $T N=I m g-S e g \cup G T$, where $I m g$ is the entire image. We define the segmentation error as:

$$
\text { error }=1-\text { accuracy }=1-\left(\frac{T P+T N}{I m g}\right)
$$

Table 1 shows comparative error results for the 17 data sets which are not used in the training with the known ground truth (manually segmented by an expert). The differences in the mean errors between the proposed segmentation, the level-set shape based approach of Tsai et al. [17], and ASM segmentation [18] are statistically significant according to the unpaired $t$-test (the two-tailed value $P$ is less than 0.0001 ).

Table 1. Accuracy of our segmentation on 17 data sets in comparison to the level sets based segmentation in [17] and the active shape model (ASM) in [18].

\begin{tabular}{|l|c|c|c|}
\cline { 2 - 4 } \multicolumn{1}{c|}{} & \multicolumn{3}{c|}{ Algorithm } \\
\cline { 2 - 4 } \multicolumn{1}{c|}{} & Our & {$[17]$} & {$[18]$} \\
\hline Minimum error, \% & $\mathbf{0 . 1 7}$ & 6.70 & 10.50 \\
Maximum error, \% & $\mathbf{2 . 1 5}$ & 14.10 & 23.30 \\
Mean error, \% & $\mathbf{1 . 3 0}$ & 9.70 & 13.98 \\
Standard deviation,\% & $\mathbf{1 . 7 0}$ & 3.30 & 7.10 \\
\hline Significant difference, P-value & & 0.0001 & 0.0001 \\
\hline
\end{tabular}




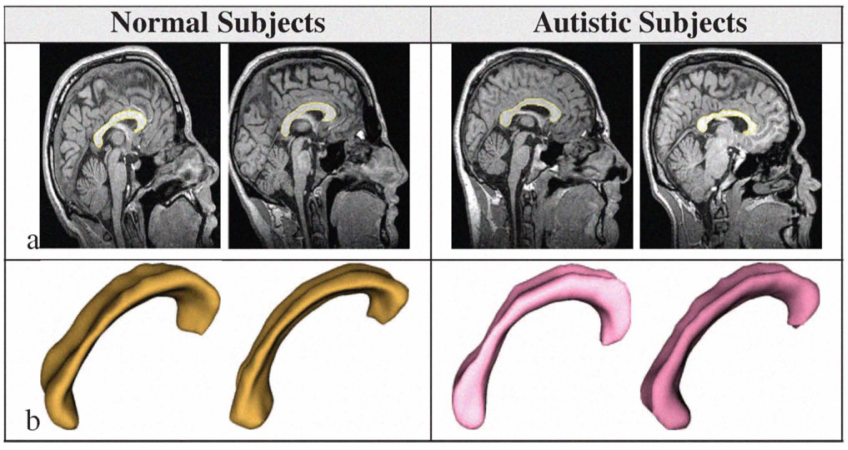

Fig. 5. Visualized 2D (a) and 3D (b) segmented CC.

Diagnostic results: The differences in the mean length of the centerline of CCs between autistic and control subjects are statistically significant according to the unpaired $t$-test (the two-tailed value $P$ is less than 0.0187) as shown in Table 2. This encourage us to use the centerline length as a discriminant feature to distinguish between autistic and normal subjects. The training subset for classification (17 persons used in the training) was arbitrarily selected among all the 34 subjects. The accuracy of classification, based on using kNearest neighbor classifier for both the training and test subjects, was evaluated using the $\chi^{2}$-test at the three confidence levels $-85 \%$, $90 \%$ and $95 \%$ - in order to examine significant differences in the Levy distances. As expected, the $85 \%$ confidence level yielded the best results - the correctly classified 16 out of 17 autistic subjects (a $94.11 \%$ accuracy), and 15 out of 17 control subjects (a $88.23 \%$ accuracy). At the $90 \%$ confidence level, 16 out of 17 autistic subjects were still classified correctly; however, only 14 out of 17 control subjects were correct, bringing the accuracy rate for the control group down to $82.35 \%$. The $95 \%$ confidence level obviously gives the smaller accuracy rates for both the groups, namely, 14 out of 17 correct answers for autistic subjects (82.35\%) and 13 out of 17 control subjects $(76.47 \%)$. The classification of the same data sets, based on traditional volumetric approach of the cerebral white matter, is 10 out of 17 autistic subjects (a $58.82 \%$ accuracy) and 11 out of 17 control subjects (a $64.71 \%$ accuracy) at an $85 \%$ confidence interval [13]; these results highlight the advantage of the proposed diagnostic approach.

Table 2. Statistical analysis for the extracted centerline length of CCs for 17 autistic subjects and 17 control subjects (All units are in $\mathrm{mm})$.

\begin{tabular}{|l|c|c|}
\cline { 2 - 3 } \multicolumn{1}{c|}{} & Autism & Control \\
\hline Minimum Centerline Length & 79.8 & 84.4 \\
Maximum Centerline Length & 93.8 & 108.8 \\
Mean Centerline Length & 88.4 & 92.8 \\
Standard Deviation & 3.9 & 6.2 \\
\hline Significant Difference, P-value & \multicolumn{2}{|c|}{0.0187} \\
\hline
\end{tabular}

In total, these preliminary results show that the centerline-based shape analysis of the $\mathrm{CC}$ is able to accurately discriminate between the autistic and normal subjects. These findings lead towards more efficient noninvasive computer assisted diagnostics of autism. In the future, we are going to investigate different brain structures in order to quantitatively characterize the development and temporal changes of an autistic brain.

\section{REFERENCES}

[1] P. Brambilla, A. Hardan, S. Nemi, "Brain anatomy and development in autism: review of MRI studies," Brain Research Bulletin, vol. 61, pp. 557-569, 2003.

[2] M. Stevens, D. Fein, M. Dunn, et al., "Subgroups of children with autism by cluster analysis: a longitudinal examination," $J$ Am Acad Child Adolesc Psychiatry, vol. 39, pp. 346-352, 2000.

[3] E. Courchesne et al., "Unusual brain growth patterns in early life in patients with autistic disorder: an mri study," Neurology, vol. 57, no. 2, pp. 245-254, 2001.

[4] R. A. Carper, P. Moses, Z. D. Tigue, and E. Courchesne, "Cerebral lobes in autism: early hyperplasia and abnormal age effects," Neuroimage, pp. 1038-1051, 2002.

[5] J. Piven, J. Bailey, B.J. Ranson, and S. Amdt, "An MRI study of the corpus callosum in autism," Am. J. Psychiatry, vol. 154, no. 8, pp. 1051-1056, 1997.

[6] F. Manes, J. Piven, D. Vrancic, V. Nanclares, C. Plebst, and S. Starkstein, "An MRI study of the corpus callosum and cerebellum in mentally retarded autistic individuals," J. Neuropsychiatry Clin Neurosci, vol. 11, no. 4, pp. 470-474, 1999.

[7] A.Y. Hardan, N.J. Minshew, and M.S. Keshavan, "Corpus callosum size in autism," Neurology, pp. 1033-1036, 2000.

[8] M. K. Chung, K. M. Dalton, A. L. Alexander, and R. J. Davidson, "Less white matter concentration in autism: 2D voxelbased morphometry," Neuroimage, 2004.

[9] Q. He, Y. Duan, J. Miles, and N. Takahashi, "Statistical Shape Analysis of the CorpusCallosum in Subtypes of Autism," Proc. 7th IEEE Int. Conf. BIBE, 14-17 Oct. 2007, pp. 1087-1091.

[10] C. N. Vidal, R. Nicolson, et al., "Mapping corpus callosum deficits in autism: An index of aberrant cortical connectivity," Biol Psychiatry, vol. 60, pp. 218-225, 2006.

[11] A. El-Baz and G. Gimelfarb, "Image segmentation with a parametric deformable model using shape and appearance priors," Proc. IEEE Conf. Computer Vision and Pattern Recognition, Anchoradge, USA, 23-28 June 2008, pp. 1-8, 2008.

[12] A. Farag, A. El-Baz, and G. Gimel'farb, "Precise segmentation of multimodal images," IEEE Transactions on Image Processing, vol. 15, no. 4, pp. 952-968, 2006.

[13] A. El-Baz and G. Gimel'farb, "EM Based approximation of empirical distributions with linear combinations of discrete Gaussians," in Proc. of IEEE Int. Conf. on Image Processing (ICIP'07), San Antonio, Texas, USA, September 16-19, 2007, vol. 4, pp. 373-376.

[14] P. Viola and W. M. Wells, "Alignment by maximization of mutual information," Proc. 5th Int. Conf. Comp. Vision, 1995.

[15] L. Cohen and R. Kimmel, "Global minimum for active contour models: A minimal path approach," Int. J. Computer Vision, vol. 24, no. 1, pp. 57-78, 1997.

[16] D. Adalsteinsson and J. Sethian,"A fast level set method for propagating interfaces," J. Computational Physics, vol. 118, no. 2, pp. 269-277, 1995.

[17] A. Tsai, A. Yezzi, W. Wells, et al., "A shape based approach to the segmentation of medical imagery using level sets," IEEE Trans. Medical Imaging, vol. 22, 2003.

[18] T. Cootes and C. Taylor, "A mixture model for representing shape variation," Image Vision Computing, pp. 567-574, 1999. 